\title{
MERCADO DE TRABALHO E O ÍNDICE FIRJAN DE DESENVOLVIMENTO MUNICIPAL: PADRÕES ESPACIAIS DOS MUNICÍPIOS DO ESTADO DO PARANÁ
}

\author{
LABOR MARKET AND THE FIRJAN INDEX OF MUNICIPALITY \\ DEVELOPMENT: SPATIAL PATTERNS FOR PARANÁ
}

MUNICIPALITIES

Rayssa Vieira Kruger*

Deise Maria Bourscheidt ${ }^{* *}$

enviado: 13 marzo 2020 - aprobado: 03 noviembre 2020

\begin{abstract}
Resumo
O estudo avaliou os padrões espaciais entre o Índice FIRJAN de desenvolvimento municipal - Emprego \& Renda, e o mercado de trabalho no ano de 2010 nos municípios paranaenses, por meio de análise do coeficiente de correlação e posterior análise espacial. A pesquisa mostrou que parte das mesorregiões Centro Ocidental, Centro Sul e Norte apresentaram padrão espacial Baixo-Baixo, indicando que o mercado de trabalho é desfavorecido e carece de uma maior geração de emprego e renda para poder se desenvolver economicamente. Já nas mesorregiões Norte central e Metropolitana de Curitiba, os clusters se classificaram como padrão Alto-Alto, apontando que nessas aglomerações de municípios, o mercado de trabalho é desenvolvido e possui uma maior geração de emprego na indústria, comércio e serviços.
\end{abstract}

Códigos JEL: C38, R11.

Kruger, R., \& Bourscheidt, D. M. (2021). Mercado de trabalho e o índice FIRJAN de desenvolvimento municipal: padrões espaciais dos municípios do Estado do Paraná. Estudios económicos, 38(77), pp. 99-117. DOI: https://doi.org/10.52292/j.estudecon.2021.1942

* Universidade Federal de Santa Maria, Brasil. ORCID: https://orcid.org/0000-0003-4017-3742.

E-mail: krugerrayssa@gmail.com

** Universidade Federal de Santa Maria, Brasil. ORCID: https://orcid.org/0000-0001-6782-8628.

E-mail: deiseufsm@hotmail.com 
Palavras-chave: Paraná. IFDM - emprego e renda, análise espacial.

\begin{abstract}
The study rated the spatial patterns between the FIRJAN Index of municipal development - Employment \& Income and the labor market in 2010 for Parana municipalities, through analysis the correlation coefficient and spatial analysis. The research showed that part of the mesoregions of the Western Center, Central South and North presented a Low-Low spatial pattern, indicating that the labor market is disadvantaged in this region and requires a greater generation of employment and income to be able to develop economically. In the mesoregions of Central and Metropolitan North of Curitiba, the clusters were classified as High-High, indicating that in these agglomerations of municipalities, the labor market is developed and has a greater generation of employment in the Industry, Commerce and Services.
\end{abstract}

JEL Codes: C38, R11.

Keywords: Paraná. IFDM - employment/income, spatialanalysis. 


\section{INTRODUÇÃO}

A economia do trabalho oferece o entendimento sobre uma série de forças e decisões que podem afetar o desempenho global da economia. Ela considera a força de trabalho como um elemento no processo de produção e envolve o estudo dos fatores que afetam a eficiência do trabalho, sua alocação em diferentes ocupações, setores e segmentos produtivos, como também os determinantes de seu pagamento (Kon, 2016). Deve-se ressaltar que as atividades criadoras de emprego são tomadas como os principais fatores determinantes do crescimento de uma região e de sua interação com as demais regiões (Alves \& de Lima, 2009, p. 2).

Para colaborar com a mensuração dessas variáveis, de modo a visualizar as mudanças no desempenho econômico e social, um dos indicadores existentesé o índice FIRJAN de desenvolvimento municipal (IFDM). O índice acompanha o desenvolvimento socioeconômico dos mais de cinco mil municípios brasileiros com base em três pilares do desenvolvimento humano: Educação, Saúde e Emprego e Renda (Firjan, 2015). No caso do estado do Paraná, a média do IFDM para os 399 municípios foi de 0.677 , no ano de 2010, indicando um desenvolvimento moderado (Marconato, 2015).

Acompanhando as mudanças em nível nacional, o estado do Paraná aumentou o seu dinamismo no decorrer dos anos 2000. A taxa de desemprego chegou a apenas $5.0 \%$ em 2010, esta informação foi divulgada pelo relatório intitulado “Os Vários Paranás", colocado a público em 2017 pelo Instituto Paranaense de Desenvolvimento Econômico e Social (IPARDES). Este relatório traz algumas subdivisões do estado, de acordo com sua importância econômica, social, institucional e ambiental. São elas: espacialidade com máxima relevância, espacialidades com elevada relevância, espacialidades com média relevância, espacialidade com mínima relevância e espacialidades socialmente críticas. Os dados apresentados neste relatório podem ajudar a compreender os resultados encontrados na presente pesquisa.

Tratando especificamente dos setores econômicos em que o mercado de trabalho está distribuído, segundo Fernandes e Cunha (2011), no estado do Paraná, o setor da agropecuária é o mais distribuído. Já as atividades industriais se concentram nas mesorregiões Norte Central e Metropolitana de Curitiba e uma parte da mesorregião Oeste. Com relação aos setores secundários e terciários, há uma interação entre eles, que por sua vez, estão correlacionadas com a urbanização, como é o caso das mesorregiões urbano-industrial, representadas também pelo Norte Central e Metropolitana de Curitiba. Já o Centro Sul, Sudoeste e Noroeste 
Paranaense, dadas as suas características geográficas e econômicas, ainda possuem muitos municípios com uma forte relação urbano-rural.

Desta forma, o presente estudo tem por objetivo analisar os padrões espaciais entre o IFDM - Emprego e Renda, e o emprego nas atividades econômicas no ano de 2010, nos municípios paranaenses. A escolha do IFDM - Emprego e Renda deve-se ao fato de que ele aborda aspectos como a geração de emprego formal, a absorção da mão de obra local, a geração de renda formal, os salários médios do emprego formal e a desigualdade (Firjan, 2015).

Além de fornecer informações para a elaboração de políticas voltadas ao perfil de cada região, o estudo contribui para o arcabouço científico, pois percebe-se a ausência de estudos no estado do Paraná que relacionem o IFDM e o mercado de trabalho. Estudos anteriores, como de Marconatoet al. (2015), De Lima et al. (2014) e Parré et al. (2016) abordaram outros índices de desenvolvimento e outras áreas geográficas, como o Índice de Desenvolvimento Humano Municipal (IDHM) e o Índice IPARDES de desenvolvimento dos municípios (IPDM).

\section{I. ÍNDICE FIRJAN DE DESENVOLVIMENTO MUNICIPAL: CLASSIFICAÇÃO E APLICAÇÕES}

OIFDM foi criado no ano de 2008, pela Federação das Indústrias do estado do Rio de Janeiro, com o objetivo de monitorar anualmente o desenvolvimento socioeconômico de uma região, considerando as diferentes realidades de sua menor divisão federativa: o município (Firjan, 2015).

O índice (geral) avalia, com igual ponderação, as três áreas de desenvolvimento humano: Emprego e Renda, Educação e Saúde. O índice varia de 0 a 1, sendo que sua classificação é apresentada da seguinte maneira: Municípios com IFDM entre 0.0 e 0.4 possuem baixo estágio de desenvolvimento; entre 0.4 e 0.6 possuem desenvolvimento regular; entre 0.6 e 0.8 são caracterizados com desenvolvimento moderado; e 0.8 e 1.0, com alto estágio de desenvolvimento (Firjan, 2015).

Para o cálculo do IFDM - Emprego e Renda foram utilizados dados daSecretaria do Trabalho, como a geração de emprego formal, absorção da mão de obra local, geração de renda formal, salários médios do emprego formal e desigualdade (Firjan, 2015). Ainda, esta dimensão tem por objetivo identificar as características econômicas dos municípios, acompanhando a movimentação e as características do mercado formal de trabalho. 
Arevisão de literatura mostra que o índice Firjan tem sido importante ferramenta de análise do desenvolvimento e do crescimento nos últimos anos. De Souza e De Oliveira (2018) utilizaram o índice Firjan para identificar as diferenças em termos de desenvolvimento humano da microrregião de Araguaína - TO, nos períodos de 2005 e 2013. Os resultados mostraram alguma evolução na qualidade de vida, contudo, apontaram também a necessidade de melhorias nas variáveis de emprego e renda.

O trabalho de Pereira e Moreira (2016) buscou avaliar se houve melhoria do IFDM Saúde, após participação dos municípios brasileiros em consórcio público intermunicipal de saúde (Cpis). Para isso, utilizaram um modelo de regressão com dados em painel logit ordenado para os períodos de 2005, 2009 e 2011, com 5118 municípios em cada ano. Os principais resultados apontaram que o Cpis influencia positivamente a probabilidade de o município mudar para níveis mais elevados do IFDM-SA.

Outro trabalho que avaliou os indicadores desagregados do Índice Firjan de desenvolvimento municipal, teve sua aplicação realizada nos Territórios da Cidadania do estado do Mato Grosso. Os autores consideraram o valor de 0.650 como adequado e, com base neste valor, constataram uma melhoria nos indicadores. Mesmo assim, a maioria dos municípios ainda apresentou resultados inferiores a 0.650 (Cócaro, Cardoso e Pereira, 2016).

\section{ANÁLISE ESPACIAL: APLICAÇÕES RECENTES}

Segundo Almeida (2012), a econometria especial é um ramo da econometria que incorpora nos modelos os chamados efeitos espaciais: a dependência espacial e a heterogeneidade espacial. Uma maneira de realizar esta análise espacial é fazendo uso da Análise Exploratória de dados Espaciais (AEDE), que permite detectar dependências espaciais, ou seja, identifica localidades que sofrem influência de localidades vizinhas (Pellenz \& Puchale, 2018).

Esta metodologia vem sendo aplicada por uma série de pesquisadores, ao longo dos anos, na tentativa de representar da melhor forma diferenças e dependências espaciais entre municípios, regiões, estados e países. Estudos anteriores mostram que a abrangência da AEDE é ampla.

O recente artigo de Maciel, Castro-Silva e de Farias buscou avaliar a distribuição espacial da incidência de Covid-19 e sua correlação com o índice de desenvolvimento humano municipal (IDHM) dos municípios do Ceará recuperando 
os dados da $15^{\mathrm{a}}$ e da $19^{\mathrm{a}}$ semana epidemiológica do ano de 2020. Entre os principais resultados, os autores observaram uma dependência espacial com correlação positiva moderada e formação de clusters do tipo alto-alto na região metropolitana de Fortaleza e municípios da região norte. Já nas regiões sul e sudeste, os clusters mostraram padrão baixo-baixo.

Destaca-se também o trabalho de Soares et al. (2019) que, por meio da AEDE, visualizaram a distribuição dos montantes gastos em saúde pública, em nível municipal no estado mineiro. Eles concluíram que houve problemas na aplicação destes recursos.

Também no estado de MG, a análise espacial permitiu verificar a distribuição dos repasses do ICMS-E e relacioná-lo ao PIB per capita e ao Índice Mineiro de Responsabilidade Fiscal (IMRF), considerando o período de 2004-2012. Entre as principais conclusões do trabalho, Sgarby et al. (2018) verificaram que em 2010 e 2012 os municípios que possuíam um IMRS maior, tinham vizinhos com mais altos repasses de ICMS-E.

Outra relação importante foi constatada por Krüger et al.(2017) que usou a análise espacial para relacionar o mercado de trabalho e o Programa Bolsa Família, nos municípios paranaenses. A região Centro Sul chamou a atenção pelo baixo nível de emprego, acompanhado por uma alta taxa de famílias beneficiárias do Bolsa Família.

A pobreza também tem sido analisada do ponto de vista espacial. Santos, Carvalho e Barreto (2017) identificaram a existência de clustersde pobreza regional, no trabalho que realizaram considerando todos os municípios do estado da Bahia, nos anos 2000 e 2010. Eles criaram rankings municipais, por meio do cálculo do Índice Municipal de Pobreza. Resumidamente pode-se dizer que eles registraram um alto índice de pobreza monetária em grande parte dos municípios baianos e nos seus vizinhos.

\section{METODOLOGIA}

O presente estudo, de natureza quantitativa, descreveuo padrão espacial entre o IFDM e as variáveis pertinentes ao mercado de trabalho dos municípios paranaenses. Foram utilizados dados secundários dos 399 municípios do estado do Paraná extraídos das bases de dados do Instituto Paranaense de Desenvolvimento Econômico e Social (IPARDES), doInstituto Brasileiro de Geografia e Estatística (IBGE) e do 
Sistema FIRJAN. As variáveis que foram utilizadas no estudo, para o ano de 2010, são: taxa de emprego nos principais setores econômicos (agropecuária, indústria, comércio e serviços), taxa de desemprego urbano e rural, e o IFDM - Emprego e Renda.

Para o cálculo da taxa de emprego nos setores econômicos, foi calculada a razão entre o número de pessoas ocupadas de cada setor e o número total de pessoas ocupadas. Já as taxas de desemprego (rural e urbana), foram calculadas pela fórmula (1), metodologia utilizada por Ipardes (2004, p. 64). Dessa maneira, foram utilizadas a PO e a PEA da área urbana e rural.

$$
\text { TAXA DE DESEMPREGO }=1-\left(\frac{P O}{P E A}\right) * 100
$$

Com base nisso, a análise dos dados se deu em três etapas: na primeira etapa foi realizada estatística descritiva das variáveis; na segunda etapa, uma análise de correlação entre a variável IFDM- Emprego e Renda e as variáveis referentes ao mercado de trabalho. Já a terceira etapa consistiu na Análise Exploratória de Dados Espaciais. As subseções a seguir detalharão estas etapas.

\section{III.1. Estatística descritiva}

O uso da estatística descritiva permitiu analisar elementos referentes às medidas de posição e dispersão como o valor máximo e mínimo das variáveis em estudo, a média de cada variável, o desvio padrão e o coeficiente de variação.

\section{2. Análise de correlação}

Segundo Hoffmann (2006), o coeficiente de correlação mostra a relação entre duas variáveis, ou seja, em uma amostra com "n" observações de duas variáveis, o coeficiente mostrará como se determina a correlação entre essas variáveis.

Para encontrar o coeficiente de correlação, foi usado o "Coeficiente de Correlação de Pearson", dado pela fórmula (2).

$$
r=\frac{\sum_{\mathrm{i}=1}^{\mathrm{n}}\left(X_{i}-\underline{X}\right)\left(Y_{i}-\underline{Y}\right)}{\sum_{\mathrm{i}=1}^{\mathrm{n}}\left(X_{i}-\underline{X}\right)^{2}\left(Y_{i}-\underline{Y}\right)^{2}}
$$


O coeficiente de correlação Pearson (r) pode variar de -1 a 1. O sinal indica direção positiva ou negativa do relacionamento e o valor sugere a força da relação entre as variáveis. Uma correlação negativa perfeita é representada pelo 1 e uma correlação positiva perfeita é representada pelo 1. Já uma correlação de valor zero indica que não há relação linear entre as variáveis (Figueiredo Filho \& Silva Junior, 2010).

Para testar a significância do coeficiente de correlação, será utilizado o seguinte teste de hipótese:

Hipótese nula: a correlação populacional é igual a zero $(\rho=0)$.

Hipótese alternativa: a correlação populacional é diferente de zero $(\rho \neq 0)$

A fórmula (3) mostra o cálculo para testar a hipótese de que não há nenhuma correlação entre as variáveis em análise. Observa-se pela fórmula que essa estatística segue a distribuição $t$ de Student com $n-2$ graus de liberdade. Para testar a hipótese, o resultado da fórmula (2) será comparado com o valor crítico da tabela $t$ de Student, respeitando o nível de significância e os graus de liberdade. Com base nisso, é preciso tomar cuidado com para não cometer o erro Tipo I, que consiste em rejeitar a Hipótese nula, sendo que a Hipótese nulaé verdadeira. Para isso, o nível de significância possui um papel importante na decisão de aceitar ou rejeitar a hipótese nula (Hoffmann, 2006).

$$
t=\frac{r \sqrt{n-2}}{\sqrt{1-r^{2}}}
$$

O coeficiente de correlação Pearson (r) foi obtido por meio do Software R, ou seja, o Software fornecerá o p-valor que indicará se a hipótese nula será rejeitada ou não, considerando um nível de significância de 5\%.

\section{III.3. Análise Exploratória de Dados Espaciais (AEDE)}

Um dos coeficientes de autocorrelação espacial existente no campo da AEDE é aEstatística I de Moran global bivariada e Estatística I de Moran local bivariada. Em relação à primeira, o interesse esteveem descobrir a existência de um padrão espacial no estado do Paraná entre duas variáveis e verificar se essa relação é positiva ou negativa. A Estatística I de Moran global bivariada é dada pela equação (4):

$$
I^{Z 1 z 2}=\frac{Z_{1}^{\prime} W Z_{2}}{Z_{1}^{\prime} z_{1}}
$$


Onde, representa uma das variáveis de interesse, representa a outra variável de interesse e representa a matriz de ponderação espacial. A matriz de pesos espaciais (W) utilizada neste estudo é a Rainha, que segundo Almeida (2012, p. 3), devido aos movimentos das peças em um Tabuleiro de xadrez, a matriz de pesos espaciais é Rainha quando "além das fronteiras com extensão diferente de zero, puderem ser considerados os vértices como contíguos, na visualização de um mapa".

Já o I de Moran local bivariado é a mesma lógica (equação 5), porém, para cada município do Paraná será calculado um I de Moran, ou seja, "somente os vizinhos da observação , definidos conforme uma matriz de pesos espaciais, são incluídos no cálculo" (Almeida, 2012, p. 132).

$$
I_{i}^{z_{1} z_{2}}=z_{1 i} W z_{2 i}
$$

Na pesquisa em questão, foi realizado o cálculo do I de Moran local bivariado apenas quando o global bivariado era significativo a um nível de significância de $5 \%$, podendo rejeitar a hipótese nula de que a autocorrelação espacial é zero. Caso seja significante, o I de Moran local será apresentado por meio de mapas com seus respectivos clusters, isto é, há uma abordagem complementar, que é o mapa de dispersão de Moran. Nele, podem aparecer quatro tipos de associação linear espacial (padrões espaciais): o padrão Alto-Alto (município com um alto valor da variável de interesse e é circundado por municípios com alto valor de outra variável), Baixo-Baixo (município com um baixo valor da variável de interesse e é circundado por municípios com baixo valor de outra variável), Alto-Baixo (município com um alto valor da variável de interesse e é circundado por municípios com baixo valor de outra variável e Baixo-Alto (município com um baixo valor da variável de interesse e é circundado por municípios com alto valor de outra variável) (Almeida, 2012).

Para observação do I de Moran, devem-se levar em consideração os coeficientes de correlação 1 e +1 . Quanto mais próximo o I for de -1 , a autocorrelação espacial será negativa, situação em que, quando as unidades espaciais obtiverem $\mathrm{z}$ elevados, são rodeadas por unidades espaciais com z baixos. E quando I estiver próximo de +1 , a autocorrelação será positiva, sendo que os dois elementos espaciais analisados serão elevados (Almeida, 2012).

Para o objetivo deste estudo, foram apresentados os valores do I de Moran Bivariado, procurando uma associação espacial entre o IFDM - Emprego e Renda e as demais variáveis sobre o mercado de trabalho. Para o uso deste método de análise, utilizar-se-á o software GeoDa. 


\section{RESULTADOS E DISCUSSÕES}

\section{IV.1. Estatística descritiva}

Antes de realizar uma análise mais profunda, é necessário obter informações básicas sobre os dados que estão sendo utilizados, com o objetivo de conhece-los. De acordo com a Tabela 1, observa-se que a média do IFDM - Emprego e Rendafoi de 0.53 , mostrando um desenvolvimento regular.

Tabela 1. Medidas de posição e dispersão das variáveis utilizadas

\begin{tabular}{|c|c|c|c|c|c|}
\hline Variáveis & Mínimo & Máximo & Média & DP & $\mathbf{C V}$ \\
\hline $\begin{array}{l}\text { Tx de emprego na } \\
\text { agropecuária (\%) }\end{array}$ & 0.03 & 71.94 & 3.79 & 5.70 & 150.2 \\
\hline $\begin{array}{l}\text { Tx de emprego } \\
\text { na indústria }(\%)\end{array}$ & 0.00 & 69.97 & 9.83 & 11.28 & 114.7 \\
\hline $\begin{array}{l}\text { Tx de emprego } \\
\text { no comércio }(\%)\end{array}$ & 0.00 & 24.91 & 6.05 & 3.62 & 59.89 \\
\hline $\begin{array}{l}\text { Tx de emprego } \\
\text { nos serviços }(\%)\end{array}$ & 5.75 & 55.95 & 12.97 & 5.38 & 41.51 \\
\hline $\begin{array}{l}\text { Tx de desemprego } \\
\text { rural }(\%)\end{array}$ & 0.00 & 24.06 & 2.48 & 2.18 & 87.92 \\
\hline $\begin{array}{l}\text { Tx de desemprego } \\
\text { urbano }(\%)\end{array}$ & 0.00 & 12.85 & 5.69 & 1.98 & 34.84 \\
\hline IFDM - Emprego e Renda & 0.20 & 0.89 & 0.53 & 0.12 & 22.75 \\
\hline
\end{tabular}

Fonte: elaborado pelos autores (2017).

O Coeficiente de Variação (CV) foi outra medida que se destacou. Entre as 7 variáveis, observa-se que 6 possuem baixa concentração em torno da média, pois apresentaram CV maior que 30\%. Porém, algumas dessas variáveis possuem $\mathrm{CV}$ muito maior quando se compara com as demais variáveis, como é o caso da taxa de emprego na agropecuária e na indústria. Logo, conclui-se que os dados apresentam alguns valores discrepantes. Apenas a variável IFDM - Emprego e Renda apresentou um CV de $22 \%$, indicando que possui média concentração em torno da média. Com base nessa análise inicial, os próximos tópicos aprofundarão 
a discussão, investigando o comportamento dessas variáveis e identificando esses valores discrepantes nos municípios paranaenses.

\section{IV.2. Análise de correlação}

Com o objetivo de analisar se existe uma relação linear entre o IFDM Emprego e Renda e as variáveis referentes ao mercado de trabalho, a Tabela 2 apresenta os valores do Coeficiente de Correlação e seus respectivos p-valores.

Tabela 2. Coeficiente de correlação de Pearson's

\begin{tabular}{lcc}
\hline $\begin{array}{c}\text { Variáveis correlacionadas com } \\
\text { o IFDM - Emprego e Renda }\end{array}$ & $\begin{array}{c}\text { Coeficiente de } \\
\text { Correlação }\end{array}$ & p-valor \\
\hline Taxa de emprego na agropecuária (\%) & 0.0352 & 0.4833 \\
Taxa de emprego na indústria (\%) & 0.5961 & 0.0000 \\
Taxa de emprego no comércio (\%) & 0.6717 & 0.0000 \\
\hline Taxa de emprego nos serviços (\%) & 0.4205 & 0.0000 \\
\hline Taxa de desemprego urbano (\%) & -0.1646 & 0.0010 \\
\hline Taxa de desemprego rural $(\%)$ & -0.0105 & 0.8338 \\
\hline
\end{tabular}

Fonte: elaborado pelos autores, 2017.

Nota-se que as variáveis taxa de emprego na agropecuária (TEA) e a taxa de desemprego rural (TDR) não foram significativas a um nível de significância de 5\%, ou seja, não há relação linear entre estas variáveis e o IFDM - Emprego e Renda. As demais variáveis foram significativas.

No que se refere à correlação entre os setores da economia e o IFDM Emprego e renda, a taxa de emprego na indústria(TEI), taxa de emprego no comércio (TEC) e a taxa de emprego nos serviços (TES) apresentaram coeficiente de correlação significativo e positivo, ou seja, as variáveis se comportam no mesmo sentido. Quanto à correlação entre as taxas de desemprego nas áreas geográficas e o IFDM - Emprego e renda, apenas a taxa de desemprego urbano foi significativa a um nível de significância de $5 \%$ e sua correlação foi negativa. 
Com base nestes aspectos analisados, as variáveis que apresentaram uma relação significativa a um nível de $5 \%$ serão abordadas no próximo tópico.

IV.4. Análise da autocorrelação espacial

A Tabela 3 mostra que as quatro variáveis que foram testadas apresentaram autocorrelação espacial com o IFDM - Emprego e Renda (IFDM-ER), ou seja, seu p-valor foi significativo a um nível de $5 \%$.

Tabela 3. Resultados da Estatística I de Moran Bivariada para o ano de 2010

Variáveis autocorrelacionadas espacialmente com o IFDM - Emprego e Renda

I de Moran $\quad$ p-valor

\begin{tabular}{lcc} 
Taxa de emprego na indústria (\%) & 0.1503 & 0.0010 \\
\hline Taxa de emprego no comércio (\%) & 0.0893 & 0.0010 \\
\hline Taxa de emprego nos serviços (\%) & 0.0840 & 0.0010 \\
\hline Taxa de desemprego urbano (\%) & -0.1275 & 0.0010 \\
\hline
\end{tabular}

Fonte: elaborado pelos autores, 2017.

Em relação à autocorrelação espacial do IFDM- Emprego e Renda com as demais variáveis, observa-se que a TEI, a TEC e a TES apresentaram Ide Moran positivo, indicando que há aglomerações de municípios (unidades espaciais) com valores altos de uma variável de interesse e também são circundados por valores altos da outra variável de interesse. O mesmo pode ocorrer com valores baixos.

Já a autocorrelação espacial do IFDM- Emprego e Renda, a TDU apresentou o I de Moran negativo, indicando que há aglomerações de municípios (unidades espaciais) com valores altos de uma variável de interesse e são circundados por valores baixos da outra variável de interesse, ou no sentido inverso.

Em virtude dos aspectos abordados, as variáveis que foram significativas a um nível de 5\% na autocorrelação espacial com o IFDM - Emprego e Renda serão representadas por meio de mapas de clusters bivariados, possibilitando uma melhor visualização dessas associações espaciais. 
Observa-se na Figura 1 que há uma aglomeração de municípios padrão Baixo - Baixo que abrange parte das mesorregiões Centro Ocidental, Centro Sul e Norte Pioneiro. Isso mostra que estes municípios possuem uma baixa taxa de emprego na indústria e baixo IFDM - Emprego Renda, padrão que é esperado, pois a Estatística I de Moran foi positiva. O Norte Pioneiro é classificado como de espacialidade mínima, o que pode ser corroborado pelo dado sobre o Valor Agregado Fiscal (VAF) no setor industrial, que correspondeu em 2007 a 1.22\% do total do estado, e em 2013, a 1.58\%. No período 2007-2013, a espacialidade àqual pertence a região Centro Sul perdeu participação no VAF Industrial, com o registro de $2.90 \%$ do VAF Industrial do estado em 2013 e sendo que em 2007 a participação foi de $3.05 \%$, compreendendo o período deste trabalho.

\section{Figura 1. Cluster IFDM-ER e TEI}

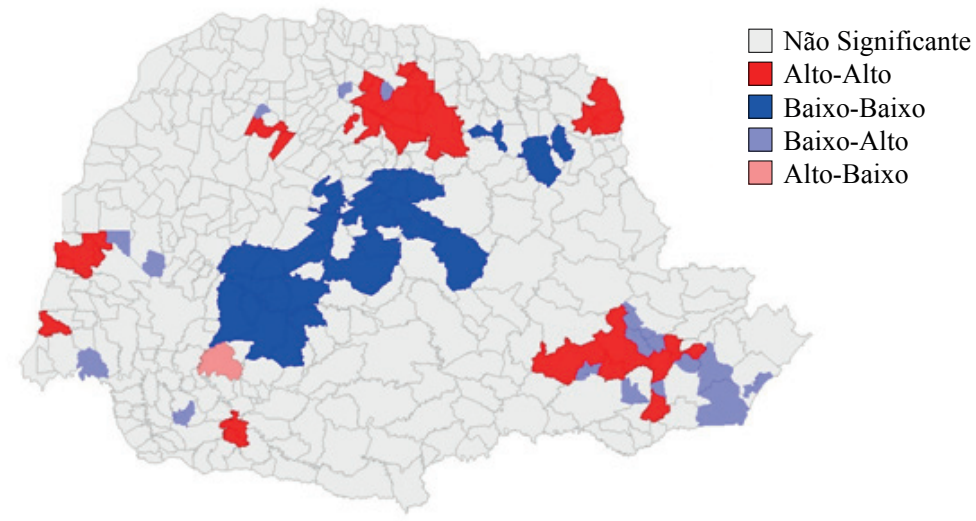

Fonte: elaborado pelos autores, 2017.

Além desse cluster padrão Baixo - Baixo, no Norte Central há uma aglomeração de municípios de padrão Alto - Alto, ou seja, municípios com alta taxa de emprego na indústria e alto IFDM - Emprego e Renda, que são os casos de alguns municípios, como Londrina (0.8374 e $10.89 \%$ ), Apucarana (0.7721 e $22.17 \%$ ), Arapongas $(0.8183$ e $30.82 \%)$, Astorga $(0.5734$ e $16.69 \%)$. Esta região teve o setor industrial como um dos mais importantes neste período (IPARDES, 2017). Na mesorregião Metropolitana de Curitiba há uma aglomeração menor, porém significativa, composta por municípios como Palmeira, Campo Largo e Curitiba, ambos com altas taxas de IFDM - Emprego e Renda e alta taxa de emprego na indústria. Essas regiões fazem parte do segundo e do primeiro espaço econômico relevante, respectivamente, ou seja, caracterizam-se como de elevada e máxima relevância 
em termos econômicos. Entre 2003 e 2013, a região metropolitana de Curitiba ampliou o número de postos de trabalho em $60 \%$, com destaque para a construção civil (IPARDES, 2017).

Observa-se na Tabela 3 que o I de Moran é positivo, logo, os padrões Baixo-Baixo e Alto-Alto predominam no mapa (Figura 2). Com base nisso, partes das mesorregiões Centro Sul e Norte Pioneiro formaram aglomerações de municípios padrão Baixo-Baixo.

Figura 2. Cluster IFDM-ER e TEC

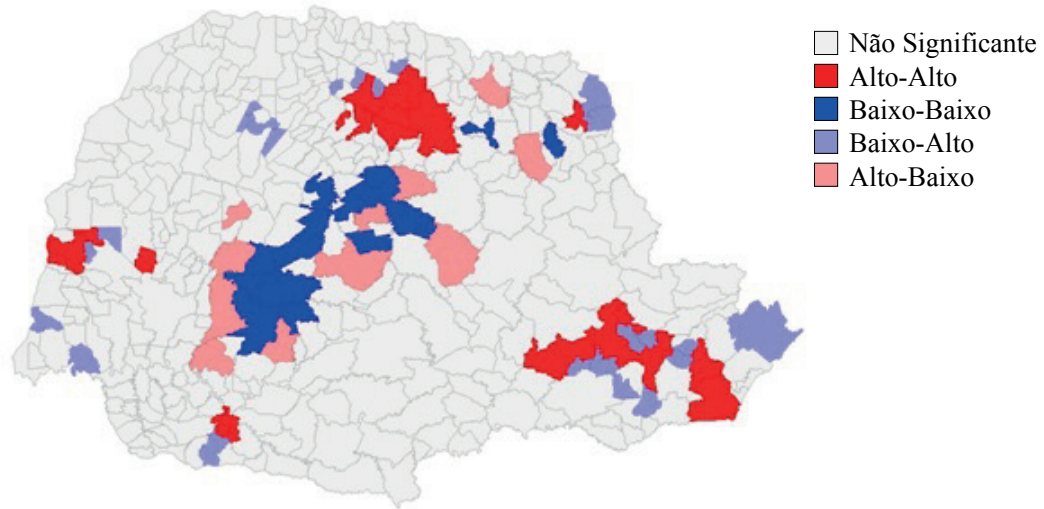

Fonte: elaborado pelos autores, 2017.

Além disso, na mesorregião Norte central e na espacialidade que inclui a região Metropolitana de Curitiba formaram-se clusters padrão Alto-Alto. Em nível estadual, o setor de comércio gerou 301077 postos de trabalho entre 2003 e 2013, sendo que deste total, 122919 foram gerados nessa espacialidade. Já na região Norte Central, o município de Maringá respondeu pela melhor participação no VAF estadual do Comércio nesse ano (5.89\%), e Londrina secundariamente, com 5.34\%, sendo que a espacialidade como um todo colaborou com 19.34\% (IPARDES, 2017).

Já na Figura 3, há uma aglomeração de municípios padrão Baixo - Baixo, que abrange parte das mesorregiões Centro Ocidental, Centro Sul e Norte Central. Essa característica indica que estes municípios possuem uma baixa taxa de emprego no setor de serviços e baixo IFDM - Emprego Renda. Além disso, entre essa aglomeração, há alguns municípios padrão Alto-Baixo, ou seja, apresentam altas taxas de emprego nos serviços, porém, baixo IFDM - Emprego e Renda, como é o caso 
de Iretama, com uma taxa de $16.46 \%$ e um IFDM - Emprego e Renda de 0.4340 . Este padrão pode acontecer em municípios pequenos, que possuem grande parte dos seus empregos no setor de serviços, em especial no serviço público, e que não conseguem desenvolver os demais setores.

Figura 3. IFDM-ER e TES

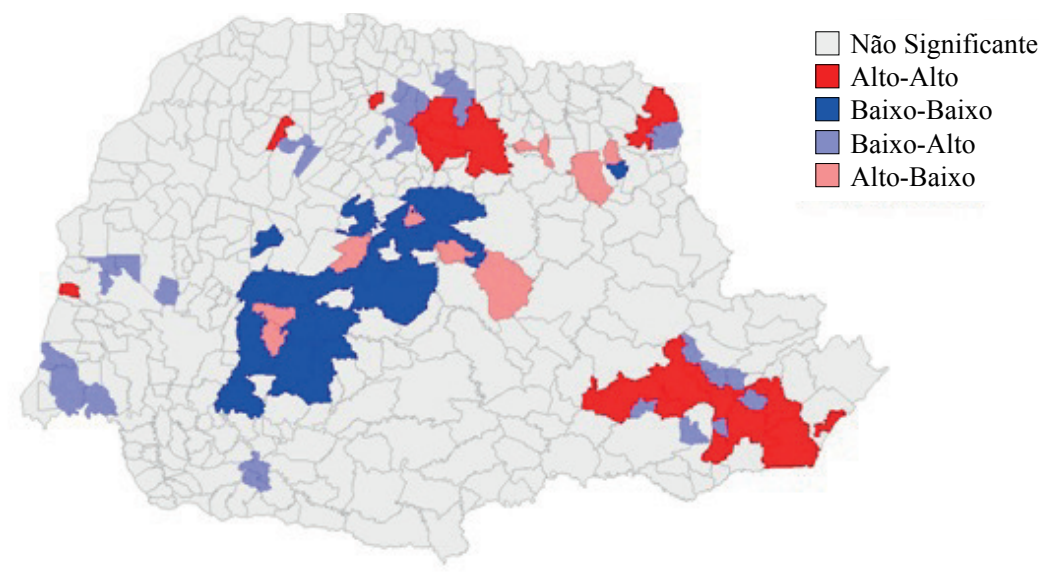

Fonte: elaborado pelos autores, 2017.

Nas mesorregiões Norte Central e na Metropolitana de Curitiba formaram-se clusterspadrão Alto-Alto, indicando que essa aglomeração de municípios possui altos valores das variáveis relativas ao emprego no setor de serviços. A região Norte Central registrou cerca de $20 \%$ do Valor Agregado Fiscal do estado no setor de serviços (IPARDES, 2017). A região metropolitana apresenta um grande número de servidores públicos, com destaque para a capital, Curitiba, sendo que os empregos formais no setor de serviços desta região correspondem a $56.86 \%$ do total do estado (IPARDES, 2017).

Assim, como ocorreu com o cluster padrão Baixo-Baixo, o padrão Alto-Alto também é acompanhado por alguns municípios que se caracterizam com padrão Baixo-Alto, como é o caso do município de Mandaguari, com uma taxa de emprego nos serviços de $10.68 \%$ e um IFDM- Emprego e Renda de 0.6528 .

Nota-se na Figura 4 que o padrão Baixo-Alto predomina nas mesorregiões Norte Central e Metropolitana de Curitiba. Característica que indica que essas aglomerações de municípios possuem baixa TDU e alto IFDM - Emprego e renda. Vale destacar que ocorreu a consolidação de parques tecnológicos na região de Curitiba no período. 


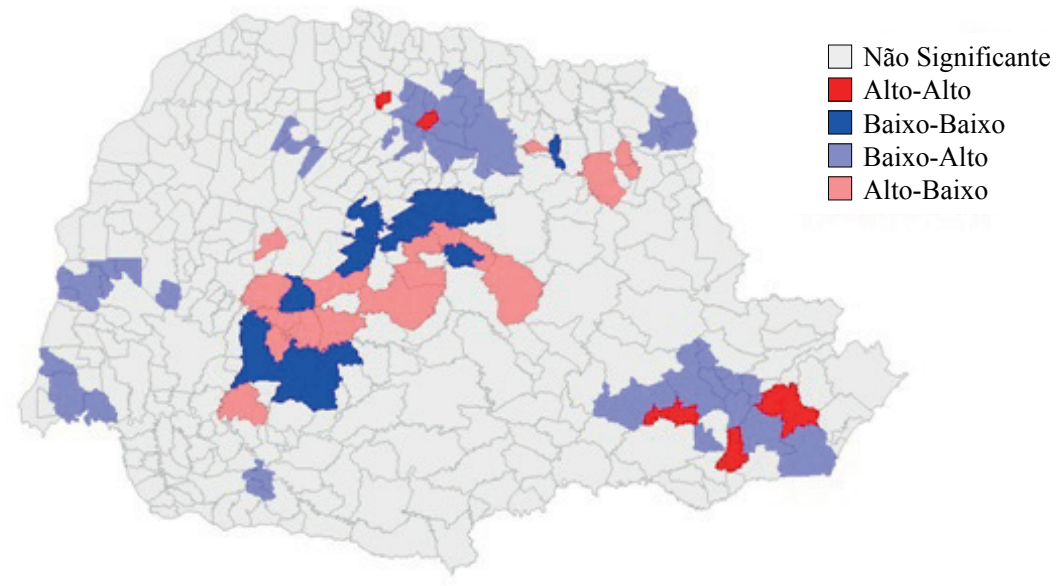

Fonte: elaborado pelos autores, 2017.

Já o padrão Alto-Baixo forma um cluster que abrange parte das mesorregiões Centro Ocidental, Centro Sul e Norte Pioneiro. Essa aglomeração de municípios apresenta altas taxas de desemprego urbano e baixo IFDM- Emprego e Renda. Vale destacar que, com relação aos anos de estudo do responsável pelo domicílio, com formação de Ensino Médio e Ensino Superior incompleto e completo, na Porção Central, no ano 2000, havia 86 municípios abaixo da média estadual. Este resultado permaneceu inalterado em 2010, e a média estadual em 2010 foi de $34.6 \%$, sendo esta uma variável relevante na determinação do nível de emprego.

\section{CONCLUSÃO}

O objetivo da presente pesquisa foi analisar os padrões espaciais entre o IFDM - Emprego e Renda e o mercado de trabalho, no ano de 2010, nos municípios paranaenses. Em síntese, o estudo mostrou que é possível rejeitar a hipótese nula de que não há autocorrelação espacial entre o IFDM - Emprego e Renda e as demais variáveis do mercado de trabalho.

Em outras palavras, o desenvolvimento do mercado de trabalho municipal no Paraná pode estar associado ao nível de industrialização de cada região do estado. Entretanto, cabe ressaltar que regiões com baixo nível de emprego na indústria podem ter sua economia baseada na agropecuária, serviços e comércio 
e, nessa questão, a agricultura familiar pode estar presente, logo, a promoção do desenvolvimento municipal por meio de políticas públicas deve estar alinhada a essas características.

Detalhadamente, notou-se que o estado do Paraná possui três principais clusters, um delespadrão Baixo-Baixo, que abrange parte das mesorregiões Centro Ocidental, Centro Sul e Norte Pioneiro, para as autocorrelações que apresentaram a Estatística I de Moran positiva, indicando que nessa região o mercado de trabalho é desfavorecido e carece de uma maior geração de emprego e renda para poder se desenvolver economicamente.

A porção central do estado possui os municípios em situação socialmente crítica, apresentando dados relativamente baixos em praticamente todas as dimensões, o que também reflete nas taxas de emprego na indústria, no comércio e nos serviços. Da mesma forma, o Norte Pioneiro tem mínima relevância no estado, tendo prejudicadas as suas taxas de emprego e, consequentemente, o seu desenvolvimento.

Já os outros dois clusters localizam-se nas mesorregiões Norte Central e Metropolitana de Curitiba, e foram caracterizados pelo padrão Alto-Alto, ou seja, nessas aglomerações de municípios, o mercado de trabalho é mais desenvolvido, principalmente porque possui uma maior geração de emprego nos setores da indústria, comércio e serviços. O desenvolvimento regional das mesorregiões metropolitanas é pautado em uma interação urbano-industrial e apresentou um padrão Alto-Alto. Em contraste, parte das mesorregiões Centro Ocidental, Centro Sul e Norte Pioneiro apresentou uma interação rural-urbana, refletindo assim em um padrão Baixo-Baixo para as atividades econômicas nessas mesorregiões.

Em relação à autocorrelação espacial do IFDM - Emprego e Renda e a TDU, os mesmos clusters se formaram, porém, com padrões diferentes. Parte das mesorregiões Centro Ocidental, Centro Sul foi caracterizada pelo padrão Alto-Baixo, ou seja, nessa região, há altas taxas de desemprego urbano, como também baixo IFDM - Emprego e Renda. É importante ressaltar, entre outros quesitos, que a taxa de escolaridade nessas regiões está abaixo da média. Já nas mesorregiões Norte Central e Metropolitana de Curitiba, formaram-se clusters padrão Baixo-Alto, ou seja, nessa região há baixas taxas de desemprego urbano, como também alto IFDM - Emprego e Renda. 
A pesquisa possibilitou uma melhor visualização dos municípios que carecem de ações que promovam o desenvolvimento do mercado de trabalho, respeitando as peculiaridades de cada região.

\section{REFERÊNCIAS BIBLIOGRÁFICAS}

Almeida, E. (2012). Econometria espacial. Alínea: Campinas-SP.

Alves, Y., \& De Lima, J. (2009). A distribuição regional do emprego formal no sul do Brasil. Textos de Economia, 11(2), 47-70. Disponível em: https://periodicos.ufsc.br/index.php/economia/article/view/2175-8085. 2008v11n2p47/10156

De Sousa, R. B., \& de Oliveira, N. M. (2018). Desenvolvimento humano da microrregião de araguaína-to: uma análise do índice firjan. Revista Geografica Academica, 12(2), 74-90.

Cócaro, H., Cardoso, R. F., \& Pereira, J. R. (2016). Territórios da Cidadania do estado de Mato Grosso: uma avaliação socioeconômica utilizando o índice FIRJAN. Interações (Campo Grande), 17(2), 193-209. Disponível em: https://doi.org/10.20435/1984042X2016204.

Programa das Nações Unidas (pnud). Atlas de desenvolvimento humano. IDHM. Brasil. Disponível em: http://www.atlasbrasil.org.br/

Firjan- Federação das Indústrias do Estado (2017). IFDM-Índice FIRJAN de Desenvolvimento Municipal:downloads. Disponível em: http://www.firjan. com.br/ifdm/downloads/

Firjan - Federação das Indústrias do Estado (2015). IFDM-Índice FIRJAN de Desenvolvimento Municipal: IFDM Edição 2010, Nota Metodológica. Disponível em: http://www.firjan.com.br/data/files/B7/43/4A/72/CE2615101BF66415F8A809C2/Anexo\%20Metodol\%C3\%B3gico\%20IFDM.pdf

Fernandes, C., \& Cunha, M. (2011). As transformações recentes no mercado de trabalho paranaense: uma aplicação do método shift-share. Revista de Economia, 37(1).

Figueiredo Filho, D., \& Junior, J. (2010). Desvendando os Mistérios do Coeficiente de Correlação de Pearson (r). Revista Política Hoje 18(1). Disponível em: https://periodicos.ufpe.br/revistas/politicahoje/article/view/3852

Hoffmann, R. (2006). Estatística para economistas (4a edição revisada e ampliada). São Paulo.

IPARDES (2004). Leituras regionais: Mesorregião Geográfica Sudoeste Paranaense - Curitiba: IPARDES; BRDE. Disponível em: http://www.ipardes. 
gov.br/index.php?pg_conteudo $=1 \&$ sistemas $=1 \&$ cod_sistema $=1 \&$ ano estudo $=2004$

IPARDES (2017). Instituto paranaense de desenvolvimento econômico e social: Base de Dados do Estado. Curitiba. Disponível em: http://www.ipardes. pr.gov.br/imp/index.php

IPARDES (2017). Instituto Paranaense de Desenvolvimento Econômico e Social: As espacialidades socioeconômico institucionais no período 2003-2015Curitiba: IPARDES.

Kon, A. (2016). A Economia do Trabalho-Qualificação e segmentação no Brasil. Brasil: Alta Books Editora.

Maciel, J. A. C., Castro-Silva, I. I., \& Farias, M. R. D. (2020). Análise inicial da correlação espacial entre a incidência de COVID-19 e o desenvolvimento humano nos municípios do estado do Ceará no Brasil. Revista Brasileira de Epidemiologia, 23, e200057. Disponível em: https://doi.org/10.1590/1980549720200057.

Marconato, M., Brambilla, M., \& Do Nascimento, S. (2015). Análise espacial do nível de desenvolvimento dos municípios do estado do Paraná. Economia \& Região, 3(1), 81-102. DOI: 10.5433/2317-627X.2015v3n1p81-

Pellenz, J. D. L., \& Puchale, C. L. (2018). Qualidade ambiental e seus condicionantes: uma análise multivariada para os municípios do rio grande do sul. Estúdios económicos, 35(71), 5-24.

Pereira, G. A., \& Moreira, T. B. D. S. (2016). A influência dos consórcios intermunicipais de saúde no índice Firjan de desenvolvimento municipal (IFDM). Planejamento e políticas públicas 46, 131-159. Disponível em: https://www. ipea.gov.br/ppp/index.php/PPP/article/view/520/387

(C) 2021 por los autores; licencia no exclusiva otorgada a la revista Estudios económicos. Este artículo es de acceso abierto y distribuido bajo los términos y condiciones de una licencia Atribución-No Comercial 4.0 Internacional (CC BY-NC 4.0) de Creative Commons. Para ver una copia de esta licencia, visite http://creativecommons.org/licenses/by-nc/4.0 
